\title{
EL DERECHO INTERNACIONAL HUMANITARIO $Y$ LOS CONFLICTOS ARMADOS INTERNOS INTERNACIONALIZADOS
}

\author{
por Dietrich Schindler
}

\section{Introducción}

Los conflictos armados internos internacionalizados son una frecuente característica de las últimas décadas. En muchas guerras civiles, han intervenido con sus ejércitos, en favor de una u otra Parte, Estados extranjeros, que han tratado de influir en el desenlace del conflicto. Varias son las causas que han motivado esa tendencia. Una de ellas es la creciente interdependencia de los Estados, lo que supone que cada guerra civil afecta a otros Estados y que, a la inversa, la actitud de otros Estados puede incidir, aunque no intervengan, en el desenlace de una guerra civil. Otra de las razones puede ser la sima ideológica mundial que divide a las naciones entre sí, lo que origina una mutua intervención parcial en conflictos internos e internacionales. Cabe mencionar, entre las demás causas, la existencia de bloques militares y de agrupaciones zonales interesados en prevenir el derrocamiento de regímenes dentro de un bloque y tendentes a fomentar trastornos en otros bloques. Otro factor que se debe tener en cuanta es la prohibición de recurrir a la fuerza en las relaciones internacionales. En épocas anteriores, los Estados hacían la guerra abierta para poder aumentar su poderío; hoy, dada la prohibición de recurrir a la fuerza, tratan más bien de lograr análogos resultados mediante la injerencia en los asuntos internos de otros Estados. La injerencia en conflictos internos es, a menudo, otra forma de guerra internacional. La inestabilidad de muchos regímenes actuales, en particular del Tercer Mundo, propicia aun más la internacionalización de conflictos internos. 
La primera guerra civil en la que hubo intervenciones a gran escala fue la española, en los años 30 . Pero sólo a partir de la Segunda Guerra Mundial hubo un mayor número de guerras civiles internacionalizadas: la guerra del Viet Nam ha quedado impresa en nuestras memorias como el más significativo ejemplo. Se debatieron, por primera vez, a raíz de dicha guerra, las cuestiones jurídicas tratadas en estas líneas. Además del conflicto del Viet Nam, también se pueden ditar como ejemplos de conflictos «mixtos » los conflictos de Hungría en 1956, del Congo en 1960, de Angola de 1960 hasta el presente, del Yemen de 1962 hasta 1970, de la República Dominicana en 1965, del Chad en varias oportunidades desde 1968, de Bangladesh (ex Pakistán oriental) en 1971, de Chipre en 1974, del Líbano desde 1976 hasta el presente, de Camboya desde 1978 hasta el presente, de Afganistán desde 1979 hasta el presente.

$\mathrm{Ni}$ en los Convenios de Ginebra ni en los de La Haya hay disposiciones concretas relativas a guerras civiles internacionalizadas, llamadas « conflictos mixtos». Tampoco se logró reglamentar, en los Protocolos adicionales de 1977, la cuestión, aunque ya se conocía bien cuando tuvo lugar la Conferencia Diplomática. Por consiguiente, el derecho aplicable en un conflicto interno internacionalizado se determina en virtud de la práctica de los Estados y de la doctrina jurídica. No es fácil encontrar respuestas, ya que el tema es muy complejo desde el punto de vista jurídico.

Durante la guerra del Viet Nam, en los años 60, había dos opiniones acerca de la aplicabilidad del derecho internacional humanitario en las guerras civiles internacionalizadas. Según la primera, una gurra civil llega a ser un conflicto armado internacional por el mero hecho de una intervención militar de Potencias extranjeras; por lo tanto, es aplicable en su totalidad el derecho internacional humanitario a todas las Partes en el conflicto, incluso al Gobierno del Estado en que estalló la guerra, y a los insurrectos. El CICR sugirió esta solución cuando, en junio de 1965, pidió a todas las Partes en el conflicto del Viet Nam que aplicasen los Convenios de Ginebra de $1949^{1}$. En lo jurídico, el señor Meyrowitz mantuvo que el derecho humanitario podía aplicarse, en su totalidad, a la guerra del Viet $\mathrm{Nam}^{2}$.

\footnotetext{
${ }^{1}$ Revista Internacional de la Cruz Roja, 1965, pág. 385 (texto francés), pág. 417 (texto inglés).

${ }^{2} \mathrm{H}$. Meyrowitz, Le droit de la guerre dans le conflit vietnamien, Annuaire français de droit international 1967, pág. 153, en particular, págs. 167-169. Traducción inglesa: The Law of War in the Vietnamese Conflict, R. A. Falk (ed.), The Vietnam War and International Law, vol. 2, Princeton 1969, pág. 516, en particular págs. 531-533.
} 
Según la otra opinión, una guerra civil internacionalizada ha de desglosarse en sus elementos internacionales y no internacionales ${ }^{3}$. Por lo que atañe a los insurrectos, su conflicto armado con el Gobierno en el poder, así como su conflicto con el Estado extranjero que ayuda a dicho Gobierno, se considera de índole no internacional. Por el contrario, cuando los oponentes en un conflicto armado son los Estados, dicho conflicto es de índole internacional. Lo mismo puede decirse de un conflicto entre el Gobierno en el poder y un Estado que interviene en favor de los insurrectos, así como de un conflicto entre dos Estados que intervienen en bandos opuestos de la guerra civil. Por ello, de los cuatro tipos de situación en que se desglosa un conflicto interno internacionalizado, se considera que dos no son internacionales y que los otros dos son internacionales. Esta solución tiene la desventaja de que pueden aplicarse distintas normas según las Partes implicadas en el conflicto.

En la Conferencia de expertos gubernamentales sobre la reafirmación y el desarrollo del derecho internacional humanitario, que se celebró en Ginebra, el año 1971, el CICR propuso la aprobación de la siguiente disposición: "Cuando en caso de conflicto armado no internacional, una u otra Parte se beneficia de la ayuda de las fuerzas armadas operantes que un tercer Estado pone a su servicio, las Partes en conflicto aplicarán el conjunto del derecho internacional humanitario aplicable en los conflictos armados internacionales » 4.

Si hubiera sido aprobada, esta disposición habría conferido análoga protección a todas las víctimas de una guerra civil internacionalizada, independientemente de la Parte a la que pertenezcan. Pero la propuesta no recibió el suficiente apoyo. La mayoría de los expertos pensaba que tal disposición podría estimular a que los insurrectos soliciten ayuda exterior con objeto de mejorar su condición jurídica ${ }^{5}$.

${ }^{3}$ Cf. D. Bindschedler-Robert, The Law of Armed Conflict, Carnegie Endowment for International Peace, Nueva York 1971, págs. 52-53; M. Bothe, Völkerrechtliche Aspekte des Angola-Konflikts, Zeitschrit für ausländiches öffentliches Recht und Völkerrecht 37 (1977), págs 590-592; J. A. Frowein, Völkerrechtliche Aspekte des VietnamKonfliktes, ibid. 27 (1967), págs 15-19; H. Meyrowitz (nota 2), Annuaire français 162, Falk (ed.) 525; A. Rosas, The Legal Status of Prisoners of War, Helsinki 1976, 283 ss.; D. Schindler, Die Anwendung der Genfer Rotkreuzabkommen seit 1949, Annuaire suisse de droit international XXII (1965), 93-98; D. Schindler, The different Types of Armed Conflicts according to the Geneva Conventians and Protocols, Compendio de los cursos de la Academia de Derecho Internacional 163 (1979) 150-151 ; R.-J. Wilhelm, Problemes relatifs à la protection de la personne humaine par le droit international dans les conflits armés ne présentant pas un caractère international, Compendio de los cursos de la Academia de Derecho Internacional 137 (1972) 356-359.

Comité Internacional de la Cruz Roja, Informe sobre los trabajos de la Conferencia de expertos gubernamentales, 1971, párrafo 284.

${ }^{5}$ Ibid. párrafo 301. 
La oposición a esta propuesta incitó al CICR a presentar, en la segunda reunión de la Conferencia de expertos, convocada el año 1972, un proyecto enmendado en el que se combinan las dos concepciones anteriormente mencionadas ${ }^{\circ}$. Según dicho proyecto, las normas humanitarias serian aplicables en su totalidad en caso de que Estados extranjeros ayuden al Gobierno en el poder o a ambas Partes en la guerra civil, pero no si los insurrectos son los únicos que reciben ayuda del exterior. Pero los expertos tampoco aprobaron dicha propuesta, ya que consideraban que todavía favorecía demasiado a los insurrectos. Por consiguiente, el CICR cejó en su empeño de introducir, en los proyectos de ambos Protocolos, disposiciones relativas a la guerra civil internacionalizada. En la Conferencia Diplomática no se planteó, en absoluto, la cuestión de la guerra civil internacionalizada. Se consolidó, en la misma, la tradicional dicotomía entre los conflictos internacionales y no internacionales. Significativamente, no obtuvo apoyo alguno la propuesta noruega de un protocolo único aplicable tanto en los conflictos internacionales como en los no internacionales ${ }^{7}$. Por consiguiente, hoy se ha de partir de la distinción que se debe hacer entre el elemento internacional y el no internacional en una guerra civil internacionalizada.

En las siguientes observaciones se tratarán, en primer lugar, las cuatro relaciones distintas en conflictos armados internos internacionalizados; después, se hablará de la situación particular de un Gobierno establecido, por la fuerza, en el poder mediante la intervención de un Estado extranjero. Se marginará la cuestión de la legalidad de la intervención extranjera en guerras civiles, por no tener relación con la cuestión de la aplicabilidad del derecho internacional humanitario.

\section{Las cuatro relaciones distintas en los conflictos armados internos internacionalizados}

Dos de las cuatro relaciones son, obviamente, de indole internacional. La primera es la que se da entre dos Estados extranjeros que intervienen en favor de las dos Partes adversas en una guerra civil 8 . Si sus fuerzas armadas se enfrentan, o si uno de esos Estados invade el territorio del

- Comité Internacional de la Cruz Roja, Informe sobre los trabajos de la Conferencia de expertos gubernamentales, 1972, vol. I, párrafo 2.332 ss.

${ }^{7}$ Ibid. 1971, párrafo $133 ; 1972$, vol. I, párrafo $0.14 \mathrm{ff}$. y 2.71. Actas de la Conferencia Diplomática sobre la Reafirmación y el Desarrollo del Derecho Internacional Humanitario Aplicable en los Conflictos Armados, Ginebra 1974-1977, Vol. VIII, págs. 205-219.

En la guerra del Viet Nam, se consideraba perteneciente a esa categoría la relación entre los Estados Unidos y Viet Nam del Norte. 
otro, es aplicable, en su totalidad, el derecho internacional humanitario para ambas Partes. Han de aplicarse todos los tratados relativos a conflictos armados en los que son Partes los Estados concernidos, así como las normas de derecho internacional consuetudinario relativas al derecho de la guerra.

La segunda relación, la que hay entre el Estado que ayuda a los insurrectos y el Gobierno en el poder, pertenece indiscutiblemente también al ámbito de las normas relativas a conflictos armados internacionales, ya que se refiere a dos sujetos de derecho internacional ${ }^{\circ}$. En dicho caso, se plantea un particular problema cuando el Estado extranjero que ayuda a los insurrectos captura a combatientes del Gobierno en el poder y los entrega a los insurrectos. En el artículo 12 del III Convenio de Ginebra, se estipula que los prisioneros de guerra sólo pueden ser trasladados de la Potencia detentora a una Potencia Parte en el Convenio y sólo cuando la Potencia dententora está segura de que aquélla está dispuesta a aplicar y puede aplicar el Convenio. Por consiguiente, los combatientes del Gobierno en el poder que hayan sido capturados, no pueden ser entregados a los insurrectos porque dichos insurrectos no son ni pueden llegar a ser Parte en los Convenios mientras continúen siendo insurrectos. Hay una disposición análoga en el artículo 45 del IV Convenio, mediante la cual se prohíbe entregar a civiles enemigos, que están en el territorio del Estado que interviene, a una Potencia que no es Parte en ese Convenio. Sin embargo, si los insurrectos vencen y derrocan al Gobierno en su Estado, ellos serán los representantes de una Parte en los Convenios de Ginebra. Así pues, habrán de serles entregados los prisioneros de guerra y las personas civiles.

La tercera relación, entre el Gobierno en el poder y los insurrectos, pertenece a la categoría de conflicto armado no internacional a la que deben aplicarse el artículo 3 de los cuatro Convenios de Ginebra y el Protocolo II, si se reúnen las correspondientes condiciones ${ }^{10}$. Sin embargo, hay varias posibilidades para que se aplique el derecho internacional humanitario en su totalidad, entre esas dos partes:

1. En el artículo 3, se insta a que las Partes en un conflicto no internacional apliquen, mediante acuerdos especiales, o mediante declaraciones unilaterales, la totalidad o parte de las otras disposiciones de los Convenios. En la guerra del Viet Nam, los Estados Unidos y Viet Nam

${ }^{\circ}$ En la guerra del Viet Nam, se consideraba perteneciente a esa categoría la relación entre Viet Nam del Norte y Viet Nam del Sur.

${ }^{10}$ En la guerra del Viet Nam, se consideraba perteneciente a esta categoría la relación entre el Gobierno de Viet Nam del Sur y el Frente de Liberación Nacional (FNL) (Vietcong). 
del Sur se declararon dispuestos a aplicar el derecho internacional humanitario con respecto al Vietcong sobre una base más amplia que la estipulada en el artículo 3.

2. De conformidad con el derecho internacional tradicional; el Gobierno en el poder puede reconocer a los insurrectos como beligerantes. En este caso, el derecho de la guerra es aplicable en su totalidad entre ese Gobierno y los insurrectos ${ }^{11}$. No obstante, no se ha dado, en muchas décadas, tal reconocimiento, con lo cual ha perdido su importancia práctica.

Se ha argüido que la aceptación de ayuda militar extranjera, por parte del Gobierno en el poder, es un reconocimiento implícito de beligerancia. Este punto de vista fue la base de la anteriormente mencionada propuesta presentada, el año 1971, por el CICR ${ }^{12}$. Pero no puede confirmarse, en absoluto, esa concepción tras la negativa reacción, en 1971 y 1972, de los expertos gubernamentales.

3. Si un Estado extranjero presta ayuda a los insurrectos, puede considerárseles como un movimiento de resistencia organizado perteneciente a ese Estado. En el párrafo 2, de la sección A del artículo 4 del III Convenio de Ginebra (que corresponde al párrafo 2 del artículo 13 del I y II Convenios), se estipula que los miembros de «movimientos de resistencia organizados, pertenecientes a una Parte en el conflicto » serán prisioneros de guerra si caen en poder del enemigo. El derecho internacional humanitario tendría que aplicarse en este caso en su totalidad ${ }^{13}$. Por lo que respecta al conflicto del Viet Nam, el señor Meyrowitz alegaba que el Vietcong podía considerarse un movimiento de resistencia perteneciente a Viet Nam del Norte ${ }^{14}$. Sin embargo, los insurrectos difícilmente se servirán de esta posibilidad, ya que desean demostrar su independencia; por lo tanto, evitarán que se les considere como perte-

11 Véase D. Schindler, State of War, Belligerency, Armed Confict, en: A. Cassese (ed.), The New Humanitarian Law of Armed Conflict, Nápoles 1979, 3, 5-6; Wilhelm (nota 3) 326-331; Ch. Zorgbibe, La guerre civile, París 1975, 36 ss.; Zorgbibe, En los origenes del reconocimiento de beligerancia, Revista Internacional de la Cruz Roja 1977, pág. 107.

12 Vẻase XXI Conferencia Internacional de la Cruz Roja, Estambul 1969. Reafirmación y desarrollo de las leyes y costumbres aplicables en los conflictos armados, Informe presentado por el CICR, pág. 116; Conferencia de expertos gubernamentales sobre la reafirmación y el desarrollo del derecho internacional humanitario aplicable en los conflictos armados, Ginebra 1971. Documentos presentados por el CICR, V, págs 17-20.

${ }^{18}$ No obstante, eso no impediría que el Gobierno en el poder juzgase a los insurrectos por crimen de alta traición o por delitos similares (artículo 85 del III Convenio de Ginebra).

14 Meyrowitz (nota 2), Annuaire Français 172, Falk (ed.) pág. 538. 
necientes a un Estado extranjero. Aunque la palabra "perteneciente " en los Convenios no se refiere a un vínculo de dependencia en el sentido jurídico, sino a una relación de facto, en el artículo 43 del Protocolo I de 1977 se estipula, en cambio, que las fuerzas armadas, Ios grupos o las unidades de una Parte en el conflicto « están colocados bajo un mando responsable de la conducta de sus subordinados ante esa Parte », lo que implica una subordinación.

4. El señor Meyrowitz esgrimió, el año 1967, un argumento jurídico para afirmar la indole internacional de la relación entre el Gobierno en el poder y los insurrectos, así como entre el Estado que interviene a favor del Gobierno en el poder y los insurrectos. Mantuvo que, a causa de la ayuda prestada por los Estados Unidos a Viet Nam del Sur, el centro de las decisiones políticas y militares en dicho conflicto se trasladó de Saigón a Washington ${ }^{15}$. En tales circunstancias, a su parecer, ya no correspondía a la realidad de los hechos la interpretación, como guerra civil, de la relación entre Saigón y el Vietcong, así como entre los Estados Unidos y el Vietcong. Sin embargo, el señor Meyrowitz no sacó todas las conclusiones posibles de su razonamiento, aunque mantuvo, con razón, que, en la cuestión de la aplicabilidad de los Convenios de Ginebra, el Vietcong no estaba obligado por los mismos.

En la práctica, parece que sólo la primera posibilidad puede llegar a concretarse.

Por último, la cuarta relación, entre un Estado que ayuda al Gobierno en el poder y los insurrectos, pertenece también a la categoría de no internacional ${ }^{16}$. Esto se explica por el hecho de que los insurrectos carecen de estatuto jurídico en derecho internacional. En principio, hay análogas posibilidades a las antes mencionadas para la aplicabilidad del derecho internacional humanitario, en su totalidad, por lo que atañe a la relación entre el Gobierno en el poder y los insurrectos:

1. Las Partes en un conflicto armado pueden concertar acuerdos en virtud del artículo 3 de los Convenios de Ginebra o formular declaraciones específicas de que aplicarán la totalidad o parte de las otras disposiciones de los Convenios.

2. Al igual que el Gobierno en el poder, el Estado que interviene puede reconocer a los insurrectos como beligerantes y, por consiguiente, aplicar el derecho de la guerra en su totalidad en sus relaciones con los insurrec-

\footnotetext{
${ }^{15}$ Meyrowitz (nota 2), Annuaire français 167, Falk (ed.) pág 531.

${ }^{16}$ En la guerra del Viet Nam, se consideraba perteneciente a esta categoría la relación entre el Vietcong y los Estados Unidos.
} 
tos. Se puede considerar a los insurrectos como un movimiento de resistencia perteneciente al Estado que los ayuda.

3. Es de destacar, sin embargo, que sería compatible con el espíritu de los Convenios y del Protocolo I que el Estado que interviene aplique el derecho internacional humanitario en su totalidad. Un Estado que interviene en el territorio de otro Estado para combatir contra los insurrectos está haciendo uso de la propia soberanía sobre los súbditos de otro Estado, incluso si lo hace invitado o autorizado por el Gobierno en el poder. Por consiguiente, se debe considerar que esta relación, que no se tomó en cuenta en las Conferencias de 1949 y de 1977, es de índole mucho más internacional que la relación entre el Gobierno en el poder y los insurrectos. Como más arriba se dice, los Estados Unidos y Viet Nam del Sur se declararon dispuestos a aplicar los Convenios de Ginebra en la guerra del Viet Nam, por lo menos en parte, y con respecto al Vietcong.

\section{Instauración forzosa de un nuevo Gobierno mediante la intervención de un Estado extranjero}

Se plantean particulares problemas cuando un Estado interviene en el territorio de otro Estado sin el asenso del Gobierno de éste para que se establezca un nuevo Gobierno que, a su vez, autorice que actúen las fuerzas armadas presentes del Estado que interviene. Tal intervención puede darse sin que se esté librando una guerra civil en el Estado donde tiene lugar. No obstante, puede estallar, como resultado de la intervención, un conflicto armado en el territorio de dicho Estado. En tal conflicto, se dan, por un lado, las dos Partes oponentes, que pueden ser el Estado que interviene y el Gobierno por éste establecido y, por otro lado, el Gobierno desplazado, otro nuevo que ocupa su lugar o incluso simples movimientos de resistencia. Un ejemplo más antiguo es el de la invasión alemana en Noruega, el año 1940, junto con la instauración, por parte de Alemania, del Gobierno Quisling. Por lo que tendremos que pensar que la invasión alemana no estaba realmente relacionada con un gran conflicto, sino que fue una incursión militar aislada a fin de instaurar en el Estado extranjero un Gobierno aceptable para el Estado que intervenía. En tal caso, se pueden distinguir tres diferentes situaciones por lo que atañe a la aplicabilidad del derecho internacional humanitario.

Supongamos, en primer lugar, que el Gobierno existente hasta entonces haya sido desplazado y que el nuevo Gobierno se haya establecido sin que haya habido conflicto armado. En tal caso, han de aplicarse las 
normas de ocupación beligerante, mientras permanezcan en el territorio del respectivo Estado las fuerzas armadas del Estado extranjero (párrafo 2 del artículo 2 de los Convenios de Ginebra). En el artículo 47 del IV Convenio de Ginebra, se estipula que las personas protegidas no perderán los beneficios debido a cambios ocurridos, como consecuencia de la ocupación, en las instituciones o en el Gobierno del Estado ocupado. Sin embargo, no persiste indefinidamente el estatuto de ocupación beligerante en tal situación. Aunque el nuevo Gobierno se haya instaurado con éxito y aunque lo reconozca también la mayoría de los Estados extranjeros, ha de considerarse, de conformidad con el principio de efectividad y dependiendo del reconocimiento explícito o implícito de la situación por parte de otras Potencias, que ya no se reúnen las condiciones para la aplicación de los Convenios de Ginebra, incluso si todavía permanecen en el territorio las fuerzas armadas del Estado Extranjero.

Supongamos, por lo que respecta a la segunda situación, que no ha desaparecido el anterior Gobierno y que, además, sigue oponiendo resistencia, tal vez en forma de guerrilla o desde un Estado extranjero ${ }^{17}$. Dicho Gobierno está implicado en un conflicto armado internacional con el Estado que interviene, dado que sigue comportándose como un Gobierno, aunque naturalmente no sea reconocido como tal por la otra Parte en el conflicto. De conformidad con el artículo 13 de los Convenios I y II, con el artículo 4 del III Convenio y con el artículo 43 del Protocolo I, se ha de tratar como combatientes a los miembros de las fuerzas armadas de un Gobierno no reconocido.

Por lo que atañe a las relaciones entre el nuevo Gobierno y el anterior, hay entre ellos un conflicto no internacional. En conjunto, puede decirse que la posición del anterior Gobierno es análoga a la del Gobierno en el poder en una guerra civil, mientras que la posición del nuevo Gobierno es similar a la de los insurrectos. Por supuesto, puede ser que cambie la situación si el anterior Gobierno no tiene posibilidad alguna de recuperar el poder y si la mayoría de los demás Estados reconoce al nuevo Gobierno establecido por el Estado que interviene. En tal caso, el Gobierno establecido por el Estado que interviene heredará la posición del Gobierno en el poder, mientras que el anterior Gobierno se verá obligado a ocupar la posición de los insurrectos.

Pasemos a analizar una tercera situación, intermedia entre las dos anteriormente mencionadas. En el supuesto de que el Estado que interviene haya logrado, mediante un ataque por sorpresa, establecer un nuevo Gobierno sin que haya habido lucha armada alguna, se formarán gradual-

${ }^{17}$ Se ha argüido que ése es el caso de Camboya, desde 1978. 
mente movimientos de resistencia que, a pesar de todo, emprenderán la lucha contra el Estado que interviene y el Gobierno por él instaurado ${ }^{18}$. En tales circunstancias, continuarán siendo aplicables las normas relativas a la ocupación beligerante mientras el Estado que interviene esté en contacto con la población civil del Estado ocupado. Por lo que respecta a los movimientos de resistencia, así como a partes del ejército oficial del Estado ocupado que entren en combate, su estatuto en derecho internacional será distinto si luchan bajo el mando de un Gobierno o de una autoridad perteneciente al Estado ocupado. Según el ejemplo del general de Gaulle en la Segunda Guerra Mundial, si se constituye un nuevo Gobierno que emprende la lucha contra la Potencia ocupante y contra el Gobierno por ésta constituido, ese nuevo Gobierno tendrá el estatuto jurídico de un Gobierno no reconocido por el adversario, en virtud del artículo 13 del I y del II Convenios de Ginebra, del artículo 4 del III Convenio y del artículo 43 del Protocolo I. Por consiguiente, es internacional el conflicto entre la Potencia ocupante y las fuerzas armadas del nuevo Gobierno.

Por el contrario, es de índole no internacional el conflicto entre el Gobierno instaurado por la Potencia ocupante y el nuevo Gobierno. En cambio, si los movimientos de resistencia luchan sin estar dirigidos por un mando político, no pueden ser considerados como ejército del Estado ocupado, ya que ninguna autoridad se responsabilizará de los mismos. Tanto el Estado que interviene como el Gobierno por él instaurado no tienen más que aplicar a éste las normas relativas a los conflictos no internacionales.

\section{Dietrich Schindler \\ Profesor en la Universidad de Zurich Miembro del CICR}

${ }^{18}$ Se ha argüido que ése es el caso de Afganistán, desde 1979. 